\title{
On a Certain Matrix Product with Specified Latent Roots
}

\author{
By H. Nagler
}

(Received 19th July 1949. Read 4th November 1949.)

1. Vajda's paper ${ }^{1}$ in this volume has suggested to the author the following problem:

Let $A$ be a $n \times m$ matrix, $m \leqq n$, let $B$ be a $m \times n$ matrix, and let $M=I-A B$, where $I$ is the unit matrix of order $n$. Given $A$, to find $B$ such that of the $n$ latent roots of $M^{\prime} M, k$ are unity, and the remaining $n-k$ are zero.

The case considered by Vajda is that in which both $A$ and $I$ - $M$ are of maximum rank $m$; the present note takes the solution a little further without, however, answering the problem completely.

2. Consider first the more stringent requirement

$$
M^{\prime} M=\left[\begin{array}{ll}
I_{k} & 0 \\
0 & 0
\end{array}\right]
$$

where $I_{k}$ is the unit matrix of order $k$, defining by its position a mode of partitioning of the $n \times n$ matrix $M^{\prime} M$. In the same mode of partitioning let

$$
M=\left[\begin{array}{ll}
m_{1} & m_{2} \\
m_{3} & m_{4}
\end{array}\right]
$$

We then have

$$
\begin{aligned}
& m_{1}^{\prime} m_{1}+m_{3}^{\prime} m_{3}=I_{k}, \\
& m_{2}^{\prime} m_{2}+m_{4}^{\prime} m_{4}=0 .
\end{aligned}
$$

If, as we shall assume, $M$ is real, (3) gives $m_{2}=0, m_{4}=0$, since both $m_{2}^{\prime} m_{2}$ and $m_{4}{ }^{\prime} m_{4}$ are non-negative definite matrices (ref. (1), p. 97, ex. 2). Hence

$$
M=\left[\begin{array}{ll}
m_{1} & 0 \\
m_{3} & 0
\end{array}\right]
$$

and $I-M$ is at least of rank $n-k$; but since it is at most of the rank of $A$, which we shall denote by $r$, where $r \leqq m$, we have $n-k \leqq r$, or $k \geqq n-r$.

\footnotetext{
' Ref. (2).
} 
3. Let $k-(n-r)=s$, where $s$ is one of the integers $0 ; 1, \ldots, r$, and put

$$
M=\left[\begin{array}{lll}
m_{11} & m_{12} & 0 \\
m_{21} & m_{22} & 0 \\
m_{31} & m_{32} & 0
\end{array}\right], \quad A=\left[\begin{array}{l}
a_{1} \\
a_{2} \\
a_{3}
\end{array}\right],
$$

where $m_{11}$ is a $(n-r) \times\left(n_{z}^{*}-r\right)$ matrix, $m_{22}$ is a $s \times s$ matrix, while the partitioning of $A$, to accord with that of $M$, is such as to let $a_{1}, a_{2}$ and $a_{3}$ have $n-r, s$ and $r-s$ rows respectively. Without loss of generality we may assume that $r$ linearly independent rows of $A$ are formed by those of $a_{2}$ and $a_{3}$. The following theorem holds:

For the existence of a solution $B$ it is necessary and sufficient for the rows of $a_{1}$ to be independent of those of $a_{3}$.

The proof is an application of a general theorem on linear equations by which there exists a solution $B$ if and only if the linear relations between the rows of $A$ hold also for the rows of $I-M$.

Assume first that the rows of $a_{1}$ depend linearly on those of $a_{2}$ and $a_{3}$, so that there is a relation of the form $a_{1}=C a_{2}+D a_{3}$, where $C$ and $D$ are matrices. Considering the last $r-s$ columns of $I-M$ we must then have $0=C \times 0+D \times I$, or $D=0$. Hence $a_{1}=C a_{2}$, which establishes the necessity of the condition. It is also sufficient, for when it is satisfied we can determine $m_{11}$ and $m_{12}$ from the condition

$$
\left[I_{n-,}-m_{11}, \quad-m_{12}\right]=C\left[-m_{21}, \quad I_{s}-m_{22}\right],
$$

where $I_{n-r}, I_{s}$ are unit matrices of the orders of their suffixes.

In order that condition (2) should also be satisfied we must further have

$$
\left[\begin{array}{ll}
m_{11} & m_{12} \\
m_{21} & m_{22}
\end{array}\right]^{\prime}\left[\begin{array}{ll}
m_{11} & m_{12} \\
m_{21} & m_{22}
\end{array}\right]+\left[\begin{array}{ll}
m_{31} & m_{32}
\end{array}\right]^{\prime}\left[\begin{array}{ll}
m_{21} & m_{32}
\end{array}\right]=\left[\begin{array}{ll}
I_{n-r} & 0 \\
0 & I_{8}
\end{array}\right] .
$$

The preceding requirements can all be seen to be satisfied if we take

$$
M=\left[\begin{array}{lll}
I_{n-r} & 0 & 0 \\
0 & I_{s} & 0 \\
0 & 0 & 0
\end{array}\right]
$$

$B$ is then obtained by solving the system of equations

$$
\left[\begin{array}{l}
a_{2} \\
a_{3}
\end{array}\right] B=\left[\begin{array}{lll}
0 & 0 & 0 \\
0 & 0 & I_{r-8}
\end{array}\right]
$$

This completes the demonstration of the sufficiency of the condition enunciated in the theorem. 
4. In this section we shall discuss the particular case where $s=0$ or $n-r=k$. Then $a_{2}$ has no rows and $a_{1}=0$. Hence we have to solve

$$
\left[\begin{array}{c}
0 \\
a_{3}
\end{array}\right] B=\left[\begin{array}{cc}
I_{n-r}-m_{1} & 0 \\
-m_{3} & I_{r}
\end{array}\right]
$$

and, by considering the rank of the matrix formed by adjoining to $A$ columns of $I-M$, we see that $I_{n-r}-m_{1}=0$. By (2) it then follows. that $m_{3}=0$, and we solve for $B$ from the system of equations $a_{3} B=\left[\begin{array}{ll}0 & I_{r}\end{array}\right]$. In this case the determination (4) of $M$ is the only possible one.

We now return to the original problem, proposed in $\S 1$, where $M$ is a matrix such that $M^{\prime} M$ has $k$ latent roots which are unity, and $n-k$ which are zero. In this case there exists an orthogonal matrix $H$ such that, in analogy with (1),

$$
H^{\prime} M^{\prime} M H=\left[\begin{array}{ll}
I_{k} & 0 \\
0 & 0
\end{array}\right] .
$$

Since $H^{\prime} H=I$ the equations for solution, $I-A B=M^{\prime} M$, are equivalent to $I-H^{\prime} A B H=H^{\prime} M^{\prime} M H$, which are of the form already solved except that where previously we had $A$ and $B$ we now have $H^{\prime} A$ and $B H$. But by a previous result a solution $B H$ exists if and only if there is a relation of the form

$$
H^{\prime} A=\left[\begin{array}{l}
0 \\
a_{3}
\end{array}\right] \text {. }
$$

If we partition $H$ by writing $H=\left[h_{1} h_{2}\right]$, when $h_{2}$ has $r$ columns, we obtain from (6) the relations

$$
\begin{gathered}
h_{2}{ }^{\prime} A=a_{3} \\
A=H\left[\begin{array}{l}
0 \\
a_{3}
\end{array}\right]=h_{2} a_{3} .
\end{gathered}
$$

Now as $a_{3}$ is of rank $r$ the matrix product $a_{3} a_{3}{ }^{\prime}$, which is of order $r \times r$, is non-singular and so possesses an inverse (ref. (1), p. 97, ex. 3).

Hence we may write $A a_{3}{ }^{\prime}=h_{2} a_{3} a_{3}{ }^{\prime}$, or $h_{2}=A a_{3}{ }^{\prime}\left(a_{3} a_{3}{ }^{\prime}\right)^{-1}$. On the other hand, $A$ being of rank $r$, let $A_{1}$ be the matrix formed by some $r$ linearly independent columns contained in $A$. Then there exists a matrix $K$ such that $A=A_{1} K$. Hence

$$
h_{2}=A_{1} K a_{3}{ }^{\prime}\left(a_{3} a_{3}\right)^{-1}=A_{1} D,
$$


say. Since $h_{2}$ and $A_{1}$ are both of rank $r$, the $r \times r$ matrix $D=K a_{3}{ }^{\prime}\left(a_{3} a_{3}{ }^{\prime}\right)^{-i}$ is also of rank $r$ and hence possesses an inverse. Combining (7) and (9) we obtain

$$
a_{3}=D^{\prime} A_{1}^{\prime} A \text {. }
$$

Now $B$ is found from the relation $H^{\prime} A B H=I-H^{\prime} M^{\prime} M H$, which, in virtue of (5) and (6), reduces to

$$
\left[\begin{array}{l}
0 \\
a_{3}
\end{array}\right] B H=\left[\begin{array}{ll}
0 & 0 \\
0 & I_{r}
\end{array}\right],
$$

or $a_{3} B H=\left[\begin{array}{ll}0 & I_{r}\end{array}\right]$, or $a_{3} B=\left[\begin{array}{ll}0 & I_{r}\end{array}\right] H^{\prime}=h_{2}{ }^{\prime}$. Substituting in this from (9) and (10) we have

$$
D^{\prime} A_{1}^{\prime} A B=D^{\prime} A_{1}^{\prime} .
$$

Since $D$, and so $D^{\prime}$, possesses an inverse this reduces finally to

$$
A_{1}^{\prime} A B=A_{1}^{\prime} \text {. }
$$

This is an assemblage of $n$ systems of $r$ equations each and $m(\geqq r)$ unknowns each; and can therefore be solved. Although $A_{1}^{\prime}$, consisting of any $r$ linearly independent rows of $A^{\prime}$, is not determinate, equations (11) are not therefore indeterminate; for any two different determinations of $A_{1}{ }^{\prime}$ can be transformed into each other by premultiplying by a non-singular matrix of order $r$; and similarly the corresponding versions of equation (11) can be transformed into each other by premultiplying its two sides by the same matrix. This premultiplication leaves the value of $B$ unchanged.

If $m=r$, or $A$ has maximum rank, $A_{1}$ is identical with $A$, and the solution for $B$ is then unique, being

$$
B=\left(A^{\prime} A\right)^{-1} A^{\prime} \text {. }
$$

\section{REFERENCES}

(1) Turnbull, H. W. and Aitken, A. C., An introduction to the theory of canonica matrices (Glasgow, 1932).

(2) Vajda, S., Proc. Edinburgh Math. Sac. (2), 10, 1న15.

25 Helenslea Avende,

LoNDON, N.W.11. 